JMB - 愿

\title{
Proton Affinity Changes Driving Unidirectional Proton Transport in the Bacteriorhodopsin Photocycle
}

\author{
Alexey Onufriev ${ }^{1}$, Alexander Smondyrev ${ }^{2}$ and Donald Bashford ${ }^{1 *}$
}

${ }^{1}$ Department of Molecular

Biology, The Scripps Research

Institute, 10550 North Torrey

Pines Road, La Jolla, CA 92037

USA

${ }^{2}$ Schrödinger Inc., 120 West

Forty-Fifth Street, 32nd Floor

Tower 45, New York, NY

10036-4041, USA

\begin{abstract}
Bacteriorhodopsin is the smallest autonomous light-driven proton pump. Proposals as to how it achieves the directionality of its trans-membrane proton transport fall into two categories: accessibility-switch models in which proton transfer pathways in different parts of the molecule are opened and closed during the photocycle, and affinity-switch models, which focus on changes in proton affinity of groups along the transport chain during the photocycle. Using newly available structural data, and adapting current methods of protein protonation-state prediction to the non-equilibrium case, we have calculated the relative free energies of protonation microstates of groups on the transport chain during key conformational states of the photocycle. Proton flow is modeled using accessibility limitations that do not change during the photocycle. The results show that changes in affinity (microstate energy) calculable from the structural models are sufficient to drive unidirectional proton transport without invoking an accessibility switch. Modeling studies for the $\mathrm{N}$ state relative to late $M$ suggest that small structural re-arrangements in the cytoplasmic side may be enough to produce the crucial affinity change of Asp96 during $\mathrm{N}$ that allows it to participate in the reprotonation of the Schiff base from the cytoplasmic side. Methodologically, the work represents a conceptual advance compared to the usual calculations of $\mathrm{p} K_{\mathrm{a}}$ using macroscopic electrostatic models. We operate with collective states of protonation involving all key groups, rather than the individualgroup $\mathrm{p} K_{\mathrm{a}}$ values traditionally used. When combined with state-to-state transition rules based on accessibility considerations, a model for nonequilibrium proton flow is obtained. Such methods should also be applicable to other active proton-transport systems.
\end{abstract}

(C) 2003 Elsevier Ltd. All rights reserved.

Keywords: bacteriorhodopsin; continuum electrostatics; photo-cycle; protonation; affinity switch

\section{Introduction}

The light-driven proton pump, bacteriorhodopsin, is a relatively small protein made up of seven membrane-spanning helices and a retinal chromophore bound to Lys216 by a Schiff-base (SB) linkage in the central part of the molecule. Absorption

Present addresses: A. Onufriev, Computer Science Department, Virginia Tech, 660 McBryde Hall, MC 0106, Blacksburg, VA 24061, USA; D. Bashford, Hartwell Center, Mail Stop 312, St. Jude Childrens Research Hospital, 332 N. Lauderdale St., Memphis, TN 38105, USA.

Abbreviations used: RG, release group; SB, Schiff Base.

E-mail address of the corresponding author: Don.Bashford@stjude.org of a light quantum by the chromophore triggers a series of retinal isomerization changes, protonation/deprotonation events and protein structural changes that comprise the bacteriorhodopsin photocycle (Figure 1) and result, under a broad range of ambient conditions, in the net transfer of one proton from the cytoplasmic to the extracellular side of the membrane.

It has long been recognized that crucial steps in the proton pumping cycle of bacteriorhodopsin are associated with protonation-state changes of a small number of key residues, and years of experimental work have identified these residues and revealed their protonation states along the cycle. ${ }^{1-3}$ At the same time, structural studies have revealed the details of the resting state (BR state) structure in increasing detail, beginning with the 

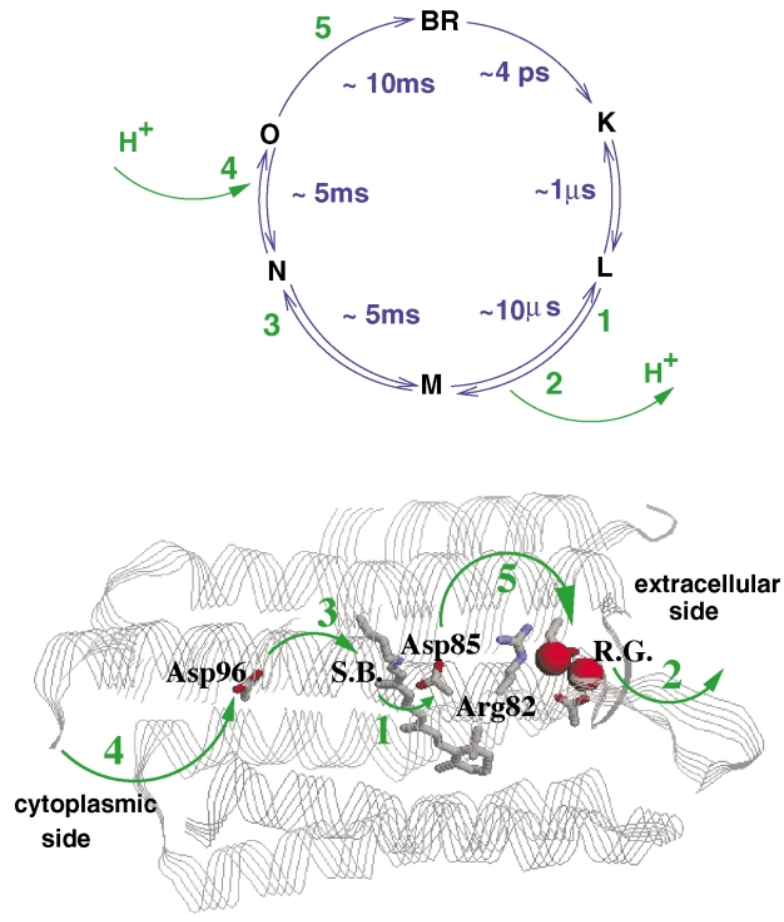

Figure 1. Conformational states in the bacteriorhodopsin photocycle (top), and key residues involved in proton transfer (bottom). Green numbers and arrows indicate proton transfer (p.t.) events. Absorption of a photon in the ground (BR) state leads to isomerization of the retinal $\mathrm{SB}$ and subsequent protein conformational changes in $\mathrm{K}$ and L. Proton transfer begins with the onset of $\mathrm{M}$ during which a proton is transferred from the SB to Asp85 (p.t. 1) and simultaneously, a different proton is released toward the extracellular side (p.t. 2). As M decays to N, SB is reprotonated from Asp96 (p.t. 3), and subsequent reprotonation of Asp96 from from the cytoplasmic side (p.t. 4) triggers the conformational change to O. Finally, in the $\mathrm{O} \rightarrow \mathrm{BR}$ transition a proton transfer from Asp85 to the $R G$ (p.t. 5) restores the original protonation state.

ground-breaking electron diffraction studies of Henderson and co-workers, ${ }^{4}$ and culminating in a number of electron diffraction and X-ray crystallographic studies of increasing resolution and completeness..$^{5-10}$ Theoretical studies ${ }^{11-16}$ utilizing this information have provided valuable insights. In the past few years, structural models of some of the intermediate states in the photocycle, based on studies of mutants, or illuminated or cryo-trapped samples, have begun to appear, ${ }^{17-26}$ setting the stage for developing a quantitative, atomistic model of the entire photo-cycle.

Here we use structural data on some of the critical intermediate states in the photocycle that have recently become available, to address one of the central questions of the field: What is the main determinant of the directionality of proton transfer? Several, not necessarily mutually exclusive hypotheses exist. ${ }^{27}$ According to one popular model, the directionality is achieved via an "accessibility switch" in which conformational changes switch the access of a key group on the pathway (e.g. the SB) from the extracellular to the cytoplasmic side, ensuring that the group can lose the proton to only one side and then re-protonate only from the other side later in the cycle. An alternative mechanism is based on the idea of "affinity switch" which attributes the directionality of transfer to changes in relative proton affinities of key groups along the pathway. For example, in the local access model of Brown et al. ${ }^{28}$ there are pathways between the SB and both Asp85 (on the extracellular side) and Asp96 (on the cytoplasmic side) that remain open throughout at least the L-to-N portion of the cycle, and the direction of proton flow depends on how affinity changes allow these groups to act as proton donors or acceptors at different stages. In the present work, a quantitative theory of how a given set of conformational changes drives proton transport is presented.

The conformational changes are, to the extent possible, taken as given from available structural studies of intermediate states, but where data are not available, or where proposed models of intermediates appear to be only partially representative, we have used modeling to fill the gaps. The energetics of possible protonation states, and thus the driving forces for possible proton-transfer events, are calculated from the structural models. Conformational changes and protonation-state changes are regarded as separate and discrete, but energetically coupled. Because the conformational states associated with photocycle intermediates have limited lifetimes, the protonation states do not necessarily fall to the lowest-energy microstate. Rather, proton exchange between pairs of groups within the protein, and between protein groups and the extracellular or cytoplasmic bulk, takes place according to microstate transition rules based on simple and intuitive considerations of whether or not a given group is readily accessible to the protons from the bulk, and whether or not there appears to be a clear proton-conducting passage between a given pair of groups inside the protein. If these rules do not change during the photocycle, the model corresponds to a pure affinity-switch model. It is shown that such a model can account for the directionality of proton transport.

\section{Results}

The relative free energies of the protonation microstates of a system with multiple interacting ionizable sites can be expressed in terms of the intrinsic $p K_{a}$ values of each site and a matrix of site-site interaction energies. The intrinsic $\mathrm{p} K_{\mathrm{a}}$, denoted $p K_{\text {intr }}$, is defined as that $p K_{a}$ that a site would have if all other sites in the protein were held in some reference state, such as the formally neutral state. ${ }^{29}$ If there were only one site, the free energy change associated with protonating the site would simply be $2.303 R T\left(\mathrm{pH}-\mathrm{p} K_{\mathrm{intr}}\right)$; and if there were multiple sites but no site-site interactions, the 
free energy to form a given microstate would be a sum of such terms. Denoting the protonation microstate of an $N$-site system by an $N$-element vector $\vec{x}$, whose elements $x_{i}$ can take on discrete values associated with the deprotonated and protonated states of their corresponding sites, the microstate free energy expression which accounts for site-site interactions is: ${ }^{30,31}$

$$
\begin{aligned}
\Delta G(\vec{x}, \mathrm{pH})= & \sum_{i} v_{i}\left(x_{i}\right) 2.303 R T\left(\mathrm{pH}-\mathrm{p} K_{\mathrm{int}, i}\right) \\
& +\frac{1}{2} \sum_{i j, i \neq j}^{N} W_{i j}\left(x_{i}, x_{j}\right)
\end{aligned}
$$

Here, $v_{i}\left(x_{i}\right)$ is the number of protons bound to site $i$ in state $x_{i}$ relative to the reference state, and $W_{i j}$ is the site-site interaction between sites $i$ and $j$ in the specified protonation states, relative to the reference state. The $\mathrm{p} K_{\text {intr }}$ and $W_{i j}$ values are calculated from model compound $\mathrm{p} K_{\mathrm{a}}$ values and the details of the protein conformation in a particular photocycle state using a macroscopic electrostatic model (see Methods).

Titratable sites in macromolecules are traditionally characterized by their apparent $p K_{\mathrm{a}}$ values which are often identified with the $\mathrm{pH}$ value at which the site is half protonated, or $\mathrm{p} K_{\text {half. The }}$ latter can be calculated ${ }^{32}$ from equation (1) by thermodynamic averaging over all possible protonation states to yield the average protonation of each site as a function of $\mathrm{pH}$. However, this traditional description and can become quite inadequate $^{33}$ when strong site-site couplings (known to occur in bacteriorhodopsin) lead to correlations and unusually shaped titration curves for individual sites, ${ }^{34,35}$ in which case $\mathrm{p} K_{\text {half }}$ may not be unambiguously defined. The more fundamental problem for the present case is that we are concerned with protonation states of deeply buried sites during short-lived structural intermediates, and an equilibrium distribution of protonation microstates might not be reached. We therefore present energetic results mainly in terms of the microstate energies of equation (1) rather than in terms of $\mathrm{p} K_{\mathrm{a}}$.

In the current work we regard as ionizable only a key subset of groups: the retinal-Lys216 SB, Asp96, Asp115, Asp85, Asp212, Arg82, Glu194 and Glu204. For the resting state (BR) conformer only, we include an $\mathrm{H}_{5} \mathrm{O}_{2}^{+}$molecule in a pocket on the extracellular side. A previous modeling study ${ }^{14}$ has proposed $\mathrm{H}_{5} \mathrm{O}_{2}^{+}$as the proton release group in accord with experimental evidence that in the BR state the release proton is stored in an hydrogenbonded water network. ${ }^{36}$ All other Asp and Glu residues are regarded as fixed in the deprotonated state, while all other Arg, Lys, and Tyr residues are regarded as fixed in the protonated state. Trial calculations have shown that these simplifications do not affect the results over a broad range of $\mathrm{pH}$ around 7.0, either because protonation microstates with contrary protonations are too high in energy,

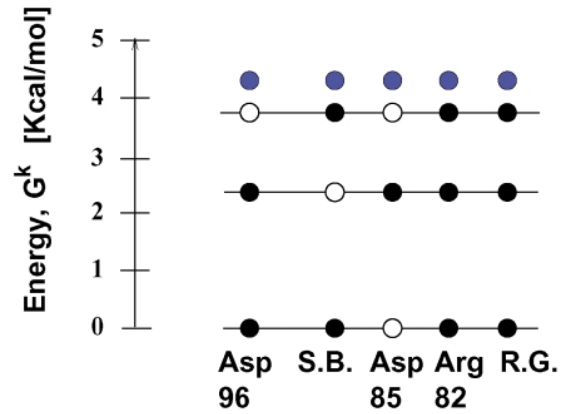

Figure 2. Ranking of the four lowest-energy protonation states of key groups for the BR conformational state of the photocycle at $\mathrm{pH}=7$. A filled/empty circle corresponds to protonated/de-protonated site. The experimentally observed "acid-blue" state of bacteriorhodopsin, which becomes the lowest energy state only at low $\mathrm{pH}$, is shown in blue. In the diagrams, Glu194, Glu204 and $\mathrm{H}_{5} \mathrm{O}_{2}^{+}$are represented collectively as the "RG", which is shown as protonated if any one of these sites is protonated; the shown energy levels are Boltzmann-averaged over the appropriate microstates. Because of the tight coupling of these three sites, states with more than one of them protonated are too high in energy to appear on the diagrams. In the BR state, it is the $\mathrm{H}_{5} \mathrm{O}_{2}^{+}$component of $\mathrm{RG}$ that holds the proton, a result consistent with previous calculations.

or because the couplings with the significant sites are too weak.

\section{BR (equilibrium) state}

The results of calculations for the first (resting) conformational state of the photocycle, the BR state, are displayed in Figure 2 as an energy level diagram; only the four lowest-ranking energy levels are shown $\dagger$. The calculations are based on the highest resolution (1.55 $\AA$ ) structure currently available. ${ }^{10}$ We have also made a comparative study in which equivalent calculations are carried out using the other high-resolution BR-state structures listed in Methods, and it was found that very similar results are obtained (unpublished results). Since the resting state is in equilibrium with respect to proton exchange with the bulk water, the lowest energy state, separated by $2.4 \mathrm{kcal} / \mathrm{mol}(\sim 4 k T$ at $300 \mathrm{~K})$ from the next highest one, is predicted as by far the predominant state. This result is consistent with the experimentally known $^{3}$ BR protonation state: Asp96, SB, Arg82 and RG protonated, and Asp85 deprotonated. It is

$\dagger$ Omitted from all the diagrams are Asp115, which is always protonated in all of the the lower-lying states (within at least $6 \mathrm{kcal} / \mathrm{mol}$ above the lowest) of the nonequilibrium intermediates, and Asp212 which is always deprotonated. While a state with Asp115 deprotonated does appear in the equilibrium BR state as the third lowest, it is still more than $3 \mathrm{kcal} / \mathrm{mol}$ above the ground protonation state and is therefore unimportant at equilibrium, and we omit it for consistency. 
also in agreement with earlier Poisson equationbased calculations of apparent $\mathrm{p} K_{\mathrm{a}}$ values in bacteriorhodopsin. ${ }^{14,34,37}$ The second-lowest state differs from the lowest only in having a proton transferred from SB to Asp85, as would happen in the $\mathrm{M}$-intermediate. The third and fourth-lowest states differ by having one fewer, and one more protons, respectively, and according to equation (1), the $\mathrm{pH}$ dependence is coupled to the total number of protons. Thus, as the ambient $\mathrm{pH}$ is lowered, the diagram changes: the third lowest state increases in relative energy while the fourth lowest decreases. This latter state, which differs from the lowestenergy state at $\mathrm{pH}=7$ only in having Asp85 protonated, corresponds to the "acid-blue" state of bacteriorhodopsin observed experimentally near $\mathrm{pH} 2$. In the present calculations, the acid-blue state becomes the lowest energy state at $\mathrm{pH} 3.7$; the experimental value ${ }^{38}$ for the $\mathrm{p} K_{\mathrm{a}}$ of the acidblue transition is 2.6. Possible sources of discrepancy include the use of the X-ray structure determined at neutral $\mathrm{pH}$ as input into the electrostatic calculations (instead of the true acid-blue state which is not available) and inherent uncertainties of the theoretical models employed here. Moreover, the experimental $\mathrm{pH}$-dependence of the purple-to-blue transition is complex, apparently involving couplings to elements of the extracellular proton-release system..$^{39,40}$ The photocycle is known to occur via the same stages ${ }^{41}$ in an extremely wide range of $\mathrm{pH}$, from at least 5 to 9 , which is larger than the typical uncertainties associated with $\mathrm{p} K_{\mathrm{a}}$ predictions based on the methodologies ${ }^{32,34}$ used here. The present work focuses on relative changes of proton affinities during the cycle, which are less sensitive to methodological uncertainties.

\section{Transition rules}

For non-equilibrium steps, we adopt the model that after a conformational change, the protonation state is initially the one that has been attained in the previous conformation, but that a re-ordering of the relative energy levels of the protonation microstates may provide a thermodynamic driving force for changes in the protonation state. However, the limited lifetime of the intermediates, and the deeply buried position of many groups involved in proton transport, imply that equilibrium with respect to protonation state may not be reached. To reflect this, we have adopted a scheme of protonation-state transition rules (Figure 3) based on the accessibility of sites to each other and to the bulk solvent. Specifically, the SB and Asp85 are separated only by one water molecule in the resting state, so proton transfer between these two is considered fast. There are also clear, short proton-transport paths connecting Asp85 to Arg82, and Arg82 to the release group (RG) complex, therefore these too are considered fast. A relatively short open pathway from Glu194/204 to the extracellular bulk allows us to assume that the proton is able to leave the $R G$

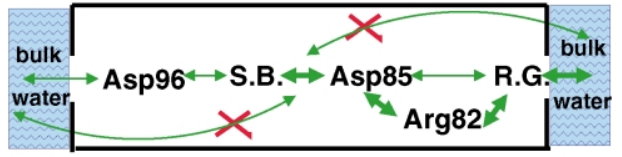

Figure 3. Model assumptions about allowable nonequilibrium proton transfers between the key ionizable groups in bacteriorhodopsin, Asp96, SB, Asp85, Arg82 and the RG, based on their proximity, apparent protontransfer pathways, and bulk solvent accessibility. Thick and thin green arrows represent fast and slow transfer paths, respectively. Asp85 and the SB cannot directly exchange protons with the bulk on the time scales involved (forbidden exchange indicated by a red X).

quickly. We also allow for a path from Asp85 to the RG that does not involve Arg82 directly, but proton transfer is assumed to be slow both because the distance is longer and because the positive charge of Arg82 would presumably raise the barrier. As for the extracellular-side pathways, the SB and Asp96 are separated by $11 \AA$ with several intervening hydrophobic side-chains and only one crystallographic water molecule (W502) wellpositioned to participate in proton transport. ${ }^{10}$ The pathway from Asp96 to the cytoplasmic bulk is similarly long and hydrophobic. These segments of the proton uptake pathway have been described as forming a hydrophobic barrier which slows proton transfer. ${ }^{42,43}$ Therefore, these two proton transfer steps are presumed to be slow. Direct proton transfers from the bulk to SB are so slow as to be considered forbidden on the time-scale of the photocycle (Figure 1); for example, when the internal proton donor Asp96 is mutated to Asn, the reprotonation of the $\mathrm{SB}$ requires tens of seconds. ${ }^{17}$

The most significant feature of these transition rules is that they do not change during the photocycle. Thus, we assume no "switch" pertaining to accessibility, and if vectorial proton transport is to occur at all within the framework of our model, it must do so via a purely "affinity switch" mechanism.

\section{Key proton transfer steps}

\section{The $M$ state}

$\mathrm{M}$ is the first intermediate in which the protonation state changes, and its energy diagram, calculated from the $M$ structure, is presented in Figure 4(A). The results are essentially the same for the two other M-state crystal structures (see Methods) used in this work. The initial microstate, which had been the lowest-energy state in the BR conformer, is now fifth from lowest in the $\mathrm{M}$ conformer. States with Asp85 protonated and/or the RG deprotonated are now lower in energy than the initial BR-like microstate, due to a movement of the positively charged Arg82 away from Asp85 and toward the RG, the moving apart of Glu194 and Glu204, both of which are negatively charged if $R G$ is deprotonated, and a weakening of the 
A

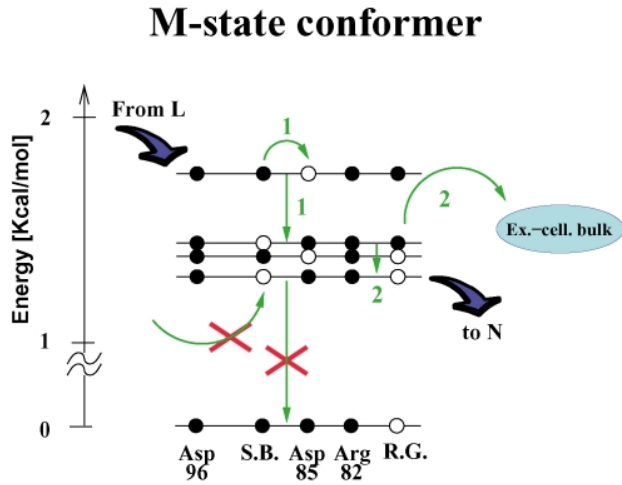

B

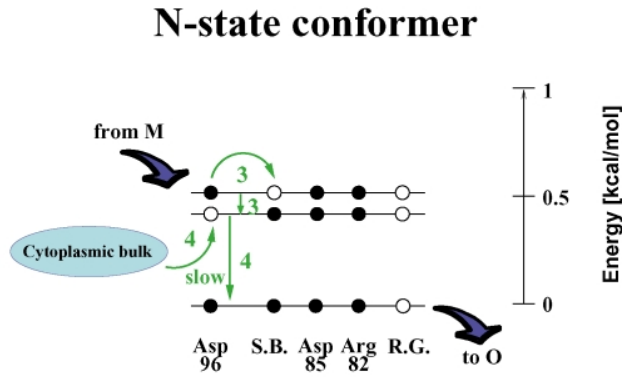

C

\section{O-state conformer}

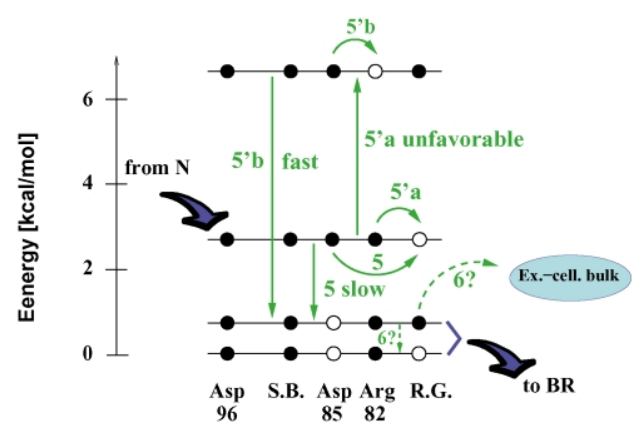

Figure 4. Protonation state diagrams of the the $\mathrm{M}, \mathrm{N}$, and $\mathrm{O}$ conformational states of the photocycle. Transitions between protonation microstates are indicated by vertical green arrows and the corresponding proton transfers by curved green arrows, both labeled using the same numbering system as Figure 1. Transitions between conformational states are indicated by thick blue arrows. (A) M-state conformation. The levels shown correspond to the M-state structure from Ref. 20 and are essentially the same for two other M-state crystal structures used in this work. The second-lowest state corresponds to the experimentally determined state, and is reachable by the transition rules, while the lowest state is inaccessible. (B) N-state conformation. Reprotonation of SB from Asp96 is favored but slow (p.t. 3), leading to the known $\mathrm{N}$ protonation state, followed by the slow reprotonation of Asp96 from the cytoplasmic bulk (p.t. 4), which triggers the conformational change to O. (C) Transitions between protonation microstates during the $\mathrm{O}$-state of the photocycle leading to the completion of the cycle and return to the BR-state. The key event is the transfer of proton from Asp85 to the RG, yielding the BR-like protonation state (second from lowest). interaction between Asp85 and the SB due to retinal isomerization and Asp85 side-chain movement. The importance of Arg82 for the proton release mechanism was noted in earlier computational and experimental studies. ${ }^{39,44}$

A proton transfer from the SB to Asp85 (p.t. 1), and the release of a proton (p.t. 2) from RG to the extracellular bulk are both allowed and fast according to the transition rules, and lead to the second lowest state. In this state, SB is deprotonated, Asp85 is protonated and the RG is empty, consistent with the experimental characterization of the late $\mathrm{M}$ state. ${ }^{3}$ The free-energy changes associated with these steps are less than $1 \mathrm{kcal} / \mathrm{mol}$, which is consistent with their observed reversibility. The lowest-energy protonation state (which is actually characteristic of the $\mathrm{O}$ state) cannot be reached directly from the second-lowest state, according to the transition rules, as that would require bringing a proton directly from the bulk to the deeply buried SB.

\section{The $N$ state}

At present, no high resolution structure of the wild-type N-state is available so we have built an atomic-level model (see Methods) starting from an available X-ray structure of $\mathrm{M} .{ }^{19}$ This late $\mathrm{M}$ structure already has features thought to be characteristic of $\mathrm{N}$, such as movements of the cytoplasmic portions of helices $F$ and $G$ to enlarge the cavities near Asp96. ${ }^{45-47}$ Molecular dynamics simulations were performed after changing to an N-like protonation state (Asp96 de-protonated) and allowing only residues near Asp96 to move significantly. Therefore, the structural model can be considered minimal in terms of differences from late $M$. The resulting structural changes are quite small and are confined to the vicinity of Asp96. In particular, we observe the reorientation of the Thr46 sidechain which is drawn closer to the Asp96 carboxylate (Figure 5).

The protonation-state energies calculated on the basis of this N-state model are shown in Figure 4(B). The N-like microstate with SB reprotonated from Asp96 has gone from being $1.25 \mathrm{kcal} / \mathrm{mol}$ higher than the M-like microstate (off the scale in Figure $4(\mathrm{~A}))$ to $0.08 \mathrm{kcal} / \mathrm{mol}$ lower. This result is consistent with experimental findings that the Mto- $\mathrm{N}$ transition involves the reversible reprotonation of the SB from Asp96. ${ }^{3}$ According to the rules of Figure 3, this transition is allowed but slow. The next lowest energy state is O-like in having Asp96 reprotonated. According to the transition rules, this state can be reached by a proton transfer from the cytoplasmic side to Asp96, but this process is expected to be slow, thus the relatively long lifetime of $\mathrm{N}$, before the reprotonation of Asp96 that is believed to trigger the change to the $\mathrm{O}$ conformer. ${ }^{48}$ Note that the lowest-energy protonation microstate in the $\mathrm{M}$ conformational state was already O-like (Figure 4(A)), but could not be reached by cytoplasmic-side proton transfer 

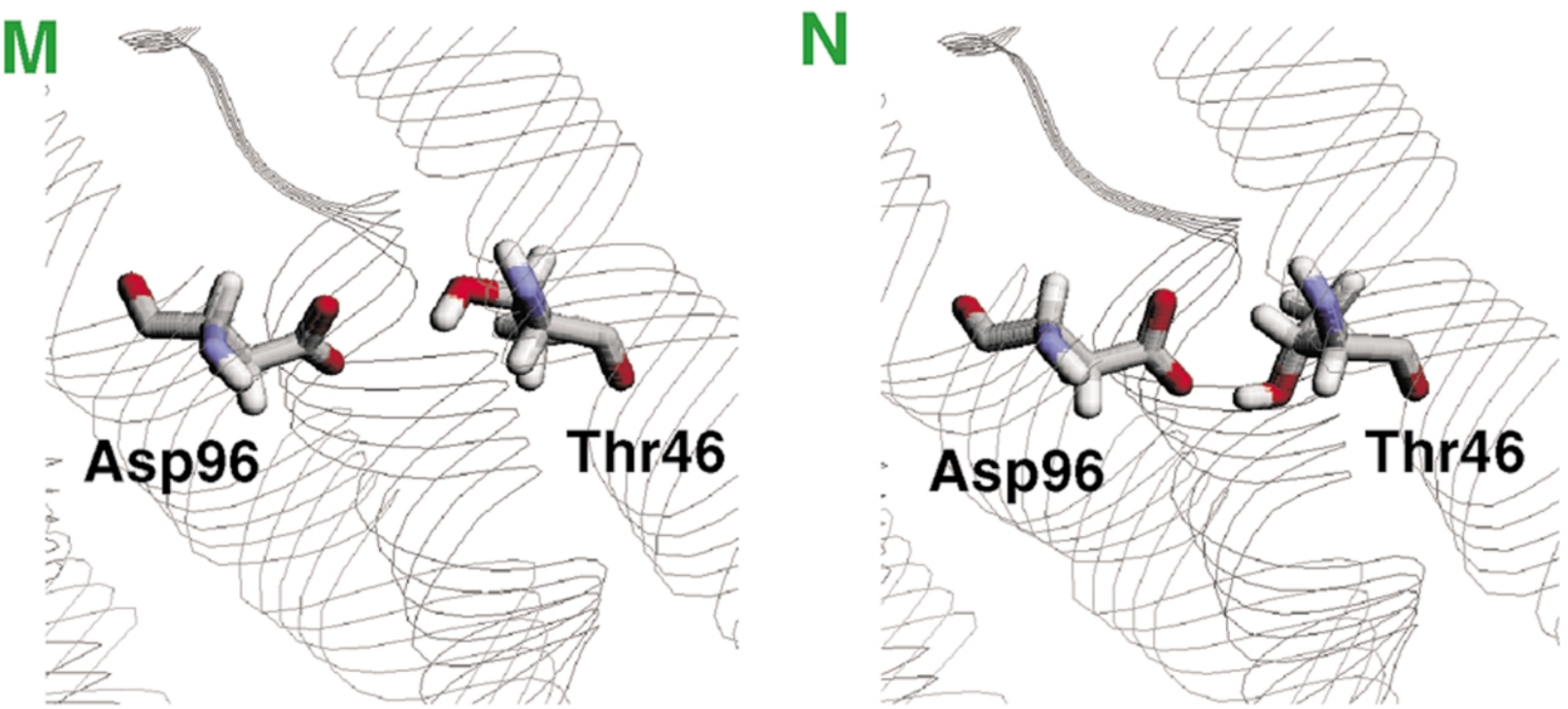

Figure 5. Structural differences between the late $\mathrm{M}$ state and our $\mathrm{N}$ state model are confined to the vicinity of the Asp96, and consist mainly of slight movement and reorientation of the Thr46 side-chain (HG-OD distance is reduced from $3.0 \AA$ in late $\mathrm{M}$ to $1.85 \AA$ in the $\mathrm{N}$ model). These result in the lowering of the calculated pK $K_{\mathrm{a}}$ of Asp96 by 5.5 units relative to the $\mathrm{M}$ state.

events, because in $\mathrm{M}$ the proton affinity of Asp96 is too high to serve as an energetically accessible element in cytoplasmic proton transfer pathway, and direct reprotonation of SB from the bulk is kinetically forbidden.

It is striking that a fairly minimal structural change (such as the Thr46 movement) leads to such a functionally significant re-ordering of the calculated protonation-state energy levels (Figure 4(A) versus Figure 4(B)); in fact our model predicts a decrease in the $\mathrm{pK}$ a of Asp96 of 5.5 units relative to the late $M$ state, in qualitative agreement with the experimental values ${ }^{49}$ of about 4. Most of the predicted drop comes from the changes in the "background" interactions (see Methods), that reflect re-distribution of charge (permanent dipoles) in the vicinity of Asp96. This explanation of the experimentally observed drop of $\mathrm{pK} K_{\mathrm{a}}$ of Asp96 represents an alternative to suggestions that the increased accessibility of the Asp96 region to water is the major factor that brings this $\mathrm{p} K_{\mathrm{a}}$ down. In this connection, we also carried out calculations on an electron microscopy structure of the resting state of a triple mutant, which has been found to have a more opened cytoplasmic side than the late M structure of Ref. 19 and has been proposed to be a model for the later stages of the photocycle, including N. ${ }^{21}$ Our results using this conformer predict a $\mathrm{p} K_{\mathrm{a}}$ of Asp96 that is too high to allow Asp96 to reprotonate the SB (energy diagram not shown). This, of course, is not to say that a structural change that considerably decreases the degree of burial of Asp96, and therefore favors its charged state, cannot bring about the drop in its $\mathrm{p} K_{\mathrm{a}}$ (through changes in the "Born term" discussed in Methods), but only that the triple-mutant structural model does not lead to such a result. In contrast, our minimal model for $\mathrm{N}$ achieves the necessary stabilization of a negative Asp96 through a change of direct electrostatic interactions with neighboring residues. Once an experimental structure of the true N-state is published, it would be an important test to use it as input for the kind of calculations presented here and compare the results.

\section{The $O$ state and completion of the cycle}

The energy-level calculations for the O-like conformer are based on a hybrid of the resting-state structure of the D85S mutant ${ }^{23}$ for the Asp85 to Asp96 region and the M-state structural model for the extracellular-side groups, Arg82 through RG, as detailed in Methods. Re-isomerization of the retinal brings the positively charged SB closer to the proton on Asp85, making this O-like protonation microstate, in which both SB and Asp85 are occupied, unfavorable (Figure $4(C)$ ). The completion of the cycle involves re-protonation of the RG from Asp85 and the return of the side-chain of Arg82 to the "up" position close to Asp85. The reprotonation can happen directly, via a slow proton transfer event (p.t. 5) over a relatively large distance between Asp85 and the RG, presumably through a water chain passing near the positively charged Arg82 (Figure 3). Alternatively, re-protonation of the RG from Asp85 could occur by an indirect, two-step route: Arg 82 would first donate a proton to the RG (p.t. 5a) and then be reprotonated by Asp85 (p.t. 5b). Although both steps are fast, the first step is uphill in energy because of the unfavorability of creating a deprotonated Arg82. Therefore, the overall process of reprotonating the RG from Asp85 would be slow by either pathway. This is consistent with the relatively long lifetime of the $\mathrm{O}$ intermediate (Figure 1). After this 
reprotonation of $\mathrm{RG}$, the O-like conformer may transiently exchange its proton with the bulk (p.t. 6 in Figure $4(\mathrm{C})$ ), but the return to the BR-like conformer and its corresponding diagram, Figure 2, makes microstates with an empty RG very unfavorable.

\section{Discussion}

Bacteriorhodopsin presents one of the best examples of a molecular machine well suited for theoretical studies: it is relatively simple and has been well characterized experimentally. Its main function, unidirectional proton transfer across the cell membrane, is known to depend critically on a small set of key titratable groups whose protonation states change during the photocycle. Calculations of the $\mathrm{p} K_{\mathrm{a}}$ values of these groups have been the subject of numerous theoretical studies, which have produced good agreement with experimental values for the equilibrium BR state of the photocycle. However, the equilibrium constants generally contain little information about the transition events that are of interest for understanding the underlying mechanisms of the photocycle. In addition, the traditional language based on of equilibrium $\mathrm{p} K_{\mathrm{a}}$ is likely to be inadequate in the case of the strongly coupled groups in bacteriorhodopsin. Here we have extended the usual methods of electrostatic models for protein $\mathrm{p} K_{\mathrm{a}}$ prediction to this non-equilibrium process by focusing on the energetics of collective protonation microstates rather than the $\mathrm{p} K_{\mathrm{a}}$ values of individual groups, and we have introduced a diagrammatic notation in which to express these results. The diagrams in themselves are suggestive of proton transfer events that may occur at various stages, and when they are combined with rules as to allowable transitions based on accessibility considerations, a model is obtained that predicts a specific sequence of proton transfer events. Applied to bacteriorhodopsin, this technique predicts vectorial proton transport, and the correct series of protonation states.

That these results are obtained with a model that does not assume any change of the accessibility of the groups during the photocycle, implies that an accessibility switch that greatly alters the protontransport connectivity between one group and another, or between groups and the bulk solution on either side is not necessary. Rather, a sufficient mechanism for vectoriality of proton transport is a pattern of changing relative energies (affinities) of protonation states during the photocycle, combined with restrictive but unchanging transition rules. A possible advantage of an affinity switch mechanism such as we present here, is that it does not require tightly fitted structural changes such as alternation between allowing or forbidding the movement of water to form transport chains between groups in one or another photocycle state. In this respect, an experimental observation that the covalent linkage between retinal and the protein is not required for the function of bacteriorhodopsin, ${ }^{50,51}$ may be more consistent with the affinity switch model being at the more fundamental level in the hierarchy of mechanisms underlying the unidirectional proton transport.

Even though the accessibility-based proton transfer rules of Figure 3 do not change during the photocycle, our model does have the effect that at certain points, protons do move in one direction and not another. This is achieved by having groups whose protonation states and proton affinities enable them (or prevent them) to act as donors or acceptors at the right time. Specifically, during $M$ the relative proton affinity of SB and Asp 85 change in a way that favors proton transfer from the former to the latter, and their close proximity means this can occur readily. The SB proton cannot move in the cytoplasmic direction, because the nearest available acceptor in that direction, Asp96, is already protonated and its high $\mathrm{p} K_{\mathrm{a}}$ in $\mathrm{M}$ discourages its transient deprotonation as part of a SB-to-cytoplasmic bulk pathway. Another possible non-productive pathway on the M-conformer energy-level diagram (Figure 4(A)) is a direct passage from the BR-like protonation state to the Olike protonation by transfer of a proton from RG to Asp85. Although this step is favorable in terms of affinities, and allowable by the rules of Figure 3, it is kinetically disfavored, since the short-distance proton transfer from SB to Asp85 will obviously be faster than the long-distance transfer from RG to Asp85. In the $\mathrm{N}$-state conformer, the relative proton affinity of Asp96 is lowered, making it energetically available to reprotonate SB from the cytoplasmic direction, while reprotonation from Asp85 (from the extracellular direction) is disfavored because the relative proton affinity of Asp85 remains too high. In these respects, the results of our calculations correspond to the "local access" proposal of Brown et al. ${ }^{28}$

Other predictions are made based on the approaches developed here. Notably, according to our calculations, small structural changes in the vicinity of Asp96 may lead to a significant decrease (in agreement with experiment) of its proton affinity in the $\mathrm{N}$ state. This represents an alternative to the previous suggestions that the opening of the cytoplasmic side in late $\mathrm{M}$ or $\mathrm{N}$ lowers the Asp96 $\mathrm{pK}_{\mathrm{a}}$ through increased hydration. ${ }^{52}$ Our calculations based on recent structural models with such openings did not lead to any decrease of the Asp96 pKa. Another observation is that transitions between the protonation microstates in the $\mathrm{M}$ and $\mathrm{N}$ states of the cycle involve very small energy changes of less than one $\mathrm{kcal} / \mathrm{mol}$ in total. This may explain the experimentally observed reversibility of the $\mathrm{M} \rightarrow \mathrm{N} \rightarrow \mathrm{O}$ transition. The conservation of energy at this stage also makes sense biologically, given the tight energy ${ }^{63}$ requirements of pumping against the transmembrane proton gradient.

The methodologies developed in this work are not confined to bacteriorhodopsin, and may be 
used to explore non-equilibrium charge transfer in other biomolecular systems.

\section{Methods}

\section{Continuum electrostatics calculations}

The continuum electrostatics methodology widely used to calculate the energetics of proton transfer is described elsewhere. ${ }^{32,34}$ The main assumption is that the shift in proton affinity of an ionizable group inside the protein relative to its value observed in free solution is due to changes in electrostatic interactions caused by the protein environment, and that these can be modeled by continuum electrostatics. Within the standard continuum approach, the protein is treated as a low dielectric medium, while the surrounding solvent is assigned a high dielectric constant. In this model the difference between a side-chain's $\mathrm{p} K_{\text {intr }}$ and the $\mathrm{p} K_{\mathrm{a}}$ of the corresponding model compound in free solution is determined by the combined effect of two distinct contributions to the total electrostatic (free) energy change. First, the "Born term" or desolvation penalty, which always penalizes burial of a charge inside a low dielectric medium. Second, the "background term" which represents the electrostatic interactions of the group in question with all other fixed charges in the molecule not belonging to any titratable groups. These energy terms, as well as the matrix of site-site interaction energies $W_{i j}$ are calculated through a sequence of numerical solutions of the Poisson-Boltzmann equation in which sites in the protein and their corresponding model compounds have their charge distributions set to those of the protonated or deprotonated form, and suitable energy differences are taken.

The $\mathrm{AMBER}^{53}$ set of partial atomic charges is used here for the protein charges. For the protonated states of Asp and Glu, in which the correct location of the proton is not known a priori, we use (except for the BR state) a "smeared charge" representation, in which the neutralizing positive charge is symmetrically distributed: 0.45 on each carbonyl oxygen atom, and 0.1 on the carbon atom. For the BR state, we use the explicit carboxylic-acid proton positions developed in our previous study, ${ }_{1}^{14}$ but we have verified that the results agree with those of our smeared-charge model. For the retinal-SB, the charges are those computed by Spassov et al. using density functional theory (DFT). ${ }^{14}$ This charge distribution might be expected to change during the all-trans $\rightarrow 13$-cis transition. However, our DFT-based calculations (manuscript in preparation) show that these changes have a relatively small effect $(\sim 0.3 \mathrm{pK})$ on the model compound $\mathrm{p} K_{\mathrm{a}}$ of the retinal in solvent. Furthermore, if the charges calculated for 13-cis configuration of the retinal found in the Mstate are used for the subsequent $\mathrm{pK}$ calculations in bacteriorhodopsin instead of the all-trans charges, the protonation-state diagram changes little. We therefore use the all-trans charge model for all of the calculations presented here. In all calculations, the interior dielectric constant, which includes the protein and lipid regions, is set to 4.0, and the exterior (solvent) dielectric constant is set to 80.0. For deeply buried groups in proteins, using an internal dielectric constant of around 4 in the continuum electrostatic calculations was found to be quite reasonable, giving good agreement with both the experiment ${ }^{54,55}$ and explicit solvent simulations. ${ }^{56}$ The Debye-Hückel screening parameter is set to correspond

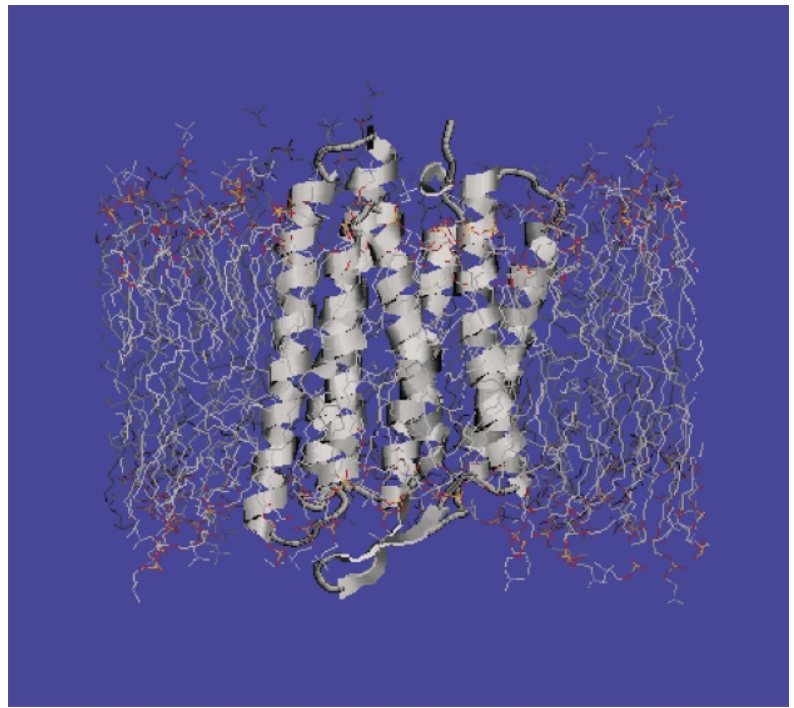

Figure 6. Bacteriorhodopsin molecule embedded in the atomic-detail membrane model.

to $0.1 \mathrm{M}$ monovalent salt concentration. The set-up and finite-difference solution of the Poisson-Boltzmann problems was carried out using the MEAD program package ${ }^{57}$ which is freely available $\dagger$. In the finite difference lattices, three levels of focusing were used, with the finest lattice having 75 grid points per edge spaced $0.5 \AA$ apart. The probe radius for defining the molecular surface, which is used as the boundary between the interior and exterior dielectric regions, is set to $1.4 \AA$.

\section{Explicit membrane representation}

An important improvement introduced here is the use of explicit atomic-level representation of the lipid membrane, Figure 6, which permits a more realistic modeling of the protein dielectric environment, compared to the more standard representation of the membrane as a uniform "slab" of low dielectric. ${ }^{34}$ The membrane environment was prepared using a method developed by one of us (A.S.) and is described in detail in a separate work (to be published). Briefly, the method incorporates any lipid molecules found in the crystal structure and builds in additional lipid by a superposition/deletion process using a modeled DPPC lipid bilayer $^{58}$ and molecular dynamics simulation. In subsequent Poisson-Boltzmann calculations, the effect of water penetration into the polar part of the membrane is mimicked by reduced head-group radii that allow some high-dielectric volumes to appear within the membrane.

All partial charges on the lipids are set to zero, including the head-groups. We find that, with a suitable choice of head-group radii, we can effectively mimic a threedielectric model, in which the protein interior and the hydrophobic regions of the membrane are assigned the dielectric constant of 4 , while the head-group region has a dielectric constant of about 20, and the bulk solvent is assigned the value of 80 . In a separate study (unpublished results) the robustness of the results with respect to variations in this membrane methodology (including radii, charges and ionic strength) was tested by a series

$\dagger$ ftp:/ftp.scripps.edu/pub/electrostatics 
of calculations on the resting state of bacteriorhodopsin, and it was found that the equilibrium ionization states of the key residues relevant to the present study were not changed, while differences in calculated $\mathrm{p} K_{\text {half }}$ remained modest.

\section{Atomic structures used}

\section{Experimental structures}

The following structures from the $\mathrm{PDB}^{59}$ were used in the study: $1 \mathrm{QHJ},{ }^{9} 1 \mathrm{C} 3 \mathrm{~W},{ }^{10} 1 \mathrm{CWQ},{ }^{19} 1 \mathrm{KGB}^{, 2}{ }^{22} 1 \mathrm{~F} 50,{ }^{20}$ $1 \mathrm{JV6}^{23}$ (BR state); $1 \mathrm{~F} 4 \mathrm{Z}^{20} 1 \mathrm{KG} 8{ }^{22}{ }^{22} \mathrm{CWQ}^{19}$ (M-state); $1 \mathrm{FBK}^{21}$ (a triple mutant having a relatively open cytoplasmic-side channel and proposed by Subramaniam et al. as a model for the N-state); $1 \mathrm{JV}^{23}$ (resting state of D85N, proposed by Rouhani et al. as an O-state model). In cases when a structure represented a mutant, the corresponding residues were changed back to those found in wild-type. In the case of $1 \mathrm{FBK}$, two calculations were performed: one with the F171C/F219L mutation as found in the structure retained, and one with these residues changed back to the WT. Both yielded the same conclusion discussed in the main text. Some structures had missing residues, in which case they were completed by taking the missing pieces from the superimposed 1QHJ structure. The superimposition was performed via RMSD fit on the back-bone atoms of major helices. Then, 100 steps of steepest descent minimization were performed to remove possible steric clashes. Only the atoms not present in the original structure were allowed to move. Hydrogen atoms were added using the LEAP module from the AMBER-7 suite of programs ${ }^{53}$ and their positions were optimized through the following three steps, during which only hydrogen atoms were allowed to move: first, 100 steps of steepest descent minimization; second, 500 steps of molecular dynamics at $300 \mathrm{~K}$, with all torsional potentials involving hydrogen atoms set to zero; and third, 100 steps of steepest decent minimization with the torsional potential switched back on. Robustness of the calculated $\mathrm{p} K_{\mathrm{a}}$ values of the $B R$ equilibrium state to protocol details, including hydrogen relaxation, was observed earlier by Spassov et al. ${ }^{14}$ All of the above simulations were performed in vacuum, with a $25 \AA$ cut-off for non-bonded interactions. The integration time-step was $2 \mathrm{fs}$, and the average temperature of the system is maintained at $300 \mathrm{~K}$ by weak coupling, via the Berendsen ${ }^{60}$ algorithm, to a heat bath with a coupling constant of $0.1 \mathrm{ps}$. We use the parm99 force-field from the AMBER-7 suite of programs, with the retinal-SB charges calculated as noted above and other energetic parameters estimated by the ANTECHAMBER module. The only exception from the above protocol is the BR-state model with the $\mathrm{H}_{5} \mathrm{O}_{2}^{+}$in the $R G$ region. This was prepared by Spassov from 1C3W PDB structure and used CHARMM ${ }^{61}$ charges; it corresponds to the "PWR" model from Ref. 14.

\section{The $N$-state model}

The N-state structural model [the PDB file is available as a Supplementary Material] was prepared by a $2.2 \mathrm{~ns}$ MD simulation at $300 \mathrm{~K}$ starting from the wild-type late $\mathrm{M}$ structure $\mathrm{PDB} 1 \mathrm{CWQ}$, in the following protonation state: Asp96 deprotonated, SB deprotonated, Asp85 deprotonated, RG deprotonated. Only the residues in the immediate vicinity of Asp96 (43-49, 93-99, and 219-224) were allowed to move during the simulation. Other details of the MD simulation protocol are the same as above, except that torsional potentials were never set to zero.

\section{The O-state model}

Our O-state model is based largely on the resting-state structure of the D85S mutant (PDB: 1JV7), which has been proposed to resemble $\mathrm{O} .^{23}$ It is not known experimentally whether the RG is actually protonated in the D85S mutant, but it is known to be protonated (as in the BR state) in the similar D85T mutant. ${ }^{62}$ In the D85S structure an extracellular release-group has Glu194 and Glu204 very close together (thus resembling the BR state), and that led to a protonated RG in preliminary calculations, contrary to the known O-state protonation of the RG which is M-like (de-protonated). We therefore assumed that the actual native $\mathrm{O}$-state should have a more M-like character for the RG, and we prepared a set of $\mathrm{p} K_{\text {intr }}$ values and a site-site interaction matrix $W_{i j}$ mostly from the values computed for the 1JV7 structure, and partly (the $\mathrm{p} K_{\text {intr }}$ values of, and site-site interactions between Glu194, Glu204, and Arg82; and the site-site interactions of Arg82 with Asp85 and SB) from the PDB $1 \mathrm{~F} 4 \mathrm{Z}$ representing the $\mathrm{M}$-state.

\section{Acknowledgements}

We thank V. Spassov, J. Lanyi, H. Luecke, J. -P. Cartailler, M. Sheves and M. Gutman for helpful discussions. A.O. thanks his former advisor, Professor D. S. Chernavskii, for valuable support and guidance, as well as for sharing his enthusiasm about the physics of bacteriorhodopsin cycle. The work was supported by NIH grant GM45607.

\section{References}

1. Rothschild, K. J. (1992). FTIR difference spectroscopy of bacteriorhodopsin: toward a molecular model. J. Bioenerg. Biomembr. 24, 147-167.

2. Haupts, U., Tittor, J. \& Oesterhelt, D. (1999). Closing in on bacteriorhodopsin: progress in understanding the molecule. Annu. Rev. Biophys. Biomol. Struct. 28, 367-399.

3. Lanyi, J. K. (2000). Molecular mechanism of ion transport in bacteriorhodopsin: insights from crystallographic, spectroscopic, kinetic, and mutational studies. J. Phys. Chem. B, 104, 11441-11448.

4. Henderson, R., Baldwin, J. M., Ceska, T. A., Zemlin, F., Beckmann, E. \& Downing, K. H. (1990). Model for the structure of bacteriorhodopsin based on high-resolution electron cryo-microscopy. J. Mol. Biol. 213, 899-929.

5. Grigorieff, N., Ceska, T. A., Downing, K. H., Baldwin, J. M. \& Henderson, R. (1996). Electron-crystallographic refinement of the structure of bacteriorhodopsin. J. Mol. Biol. 259, 393-421.

6. Pebay-Peyroula, E., Rummel, G., Rosenbusch, J. P. \& Landau, E. M. (1997). X-ray structure of bacteriorhodopsin at 2.5 Angstroms from microcrystals grown in lipidic cubic phases. Science, 277, 1676-1681.

7. Luecke, H., Richter, H.-T. \& Lanyi, J. K. (1998). Proton transfer pathways in bacteriorhodopsin at 2.3 Ångstrom resolution. Science, 280, 1934-1937. 
8. Essen, L.-O., Siegert, R., Lehmann, W. D. \& Oesterhelt, D. (1998). Lipid patches in membrane protein oligomers: crystal structure of the bacteriorhodopsinlipid complex. Proc. Natl Acad. Sci. 95, 11673-11678.

9. Belrhali, H., Nollert, P., Royant, A., Menzel, C., Rosenbusch, J. P., Landau, E. M. \& Pebay-Peyroula, E. (1999). Protein, lipid and water organization in bacteriorhodopsin crystals: a molecular view of the purple membrane at 1.9 å resolution. Struct. Fold. Des. 7, 909-917.

10. Luecke, H., Schobert, B., Richter, H.-T., Cartailler, J.-P. \& Lanyi, J. K. (1999). Structure of bacteriorhodopsin at $1.55 \AA$ resoultion. J. Mol. Biol. 291, 899-911.

11. Schulten, K., Humphrey, W., Logunov, I., Sheves, M. \& Xu, D. (1995). Molecular dynamics studies of bacteriorhodopsin's photocycles. Isr. J. Chem. 35, 447-464.

12. Scharnagl, C., Hettenkofer, J. \& Fischer, S. F. (1995). Electrostatic and conformational effects on the proton translocation steps in bacteriorhodopsin: analysis of multiple $\mathrm{M}$ structures. J. Phys. Chem. 99, 7787-7800.

13. Scharnagl, C. \& Fischer, S. F. (1996). Conformational flexibility of arginine- 82 as source for the heterogeneous and $\mathrm{pH}$-dependent kinetics of the primary proton transfer step in the bacteriorhodopsin photocycle: an electrostatic model. Chem. Phys. 212, 231-246.

14. Spassov, V. Z., Luecke, H., Gerwert, K. \& Bashford, D. (2001). $\mathrm{p} K_{\mathrm{a}}$ calculations suggest storage of an excess proton in a hydrogen-bonded water network in bacteriorhodopsin. J. Mol. Biol. 312, 203-219.

15. Baudry, J., Tajkhorshid, E., Molnar, F., Phillips, J. \& Schulten, K. (2001). Molecular dynamics study of bacteriorhodopsin and the purple membrane. J. Phys. Chem. B, 105, 905-918.

16. Hayashi, S., Tajkhorshid, E. \& Schulten, K. (2002). Structural changes during the formation of early intermediates in the bacteriorhodopsin photocycle. Biophys. J. 83, 1281-1297.

17. Luecke, H., Schobert, B., Richter, H.-T., Cartailler, J. P. \& Lanyi, J. K. (1999). Structural changes in bacteriorhodopsin during ion transport at $2 \AA$ resolution. Science, 286, 255-261.

18. Edman, K., Nollert, P., Royant, A., Belrhali, H., Pebay-Peyroula, E., Hajdu, J. et al. (1999). Highresolution X-ray structure of an early intermediate in the bacteriorhodopsin photocycle. Nature, 401, $822-826$.

19. Sass, H., Buldt, G., Gessenich, R., Hehn, D., Neff, D., Schlesinger, R. et al. (2000). Structural alterations for proton translocation in the $\mathrm{m}$ state of wild-type bacteriorhodopsin. Nature, 406, 649-653.

20. Luecke, H., Schobert, B., Richter, H.-T., Cartailler, J. P. \& Lanyi, J. K. (2000). Coupling photoisomerization of retinal to directional transport in bacteriorhodopsin. J. Mol. Biol. 300, 1237-1255.

21. Subramaniam, S. \& Henderson, R. (2000). Molecular mechanism of vectorial proton translocation by bacteriorhodopsin. Nature, 406, 653-657.

22. Facciotti, M., Rouhani, S., Burkard, F., Betancourt, F., Downing, K., Rose, R. et al. (2001). Structure of an early intermediate in the $\mathrm{m}$-state phase of the bacteriorhodopsin photocycle. Biophys. J. 81, 3442-3455.

23. Rouhani, S., Cartailler, J., Facciotti, M., Walian, P., Needleman, J., Lanyi, R. et al. (2001). Crystal structure of the d85s mutant of bacteriorhodopsin: model of an o-like photocycle intermediate. J. Mol. Biol. 313, 615-628.

24. Schobert, B., Cupp-Vickery, J., Hornak, V., Smith, S. O. \& Lanyi, J. K. (2002). Crystallographic structure of the $\mathrm{K}$ intermediate of bacteriorhodopsin: conservation of free energy after photoisomerization of the retinal. J. Mol. Biol. 321, 715-726.

25. Lanyi, J. K. \& Schobert, B. (2002). Crystallographic structure of the retinal and the protein after deprotonation of the Schiff base: the switch in the bacteriorhodopsin photocycle. J. Mol. Biol. 321, 727-737.

26. Lanyi, J. K. \& Schobert, B. (2003). Mechanism of proton transport in bacteriorhodopsin from crystallographic structures of the $\mathrm{K}, \mathrm{L}, \mathrm{M}_{1}, \mathrm{M}_{2}$ and $\mathrm{M}_{2}^{\prime}$ intermediates of the photocycle. J. Mol. Biol. 328, 439-450.

27. Lanyi, J. K. \& Luecke, H. (2001). Bacteriorhodopsin. Curr. Opin. Struct. Biol. 11, 415-419.

28. Brown, L. S., Dioumaev, A. K., Needleman, R. \& Lanyi, J. K. (1998). Connectivity of the retinal Schiff base to $\mathrm{Asp}^{85}$ and $\mathrm{Asp}^{96}$ during the bacteriorhodopsin photocycle: the local-access model. Biophys. J. 75, 1455-1465.

29. Tanford, C. \& Kirkwood, J. G. (1957). Theory of protein titration curves. I. General equations for impenetrable spheres. J. Am. Chem. Soc. 79, 5333-5339.

30. Edsall, J. T. \& Wyman, J. (1958). Biophysical Chemistry, vol. 1, Academic Press, New York.

31. Bashford, D. \& Karplus, M. (1991). Multiple-site titration curves of proteins: an analysis of exact and approximate methods for their calculation. J. Phys. Chem. 95, 9556-9561.

32. Bashford, D. \& Karplus, M. (1990). $\mathrm{pK} \mathrm{a}_{\mathrm{a}}$ s of ionizable groups in proteins: atomic detail from a continuum electrostatic model. Biochemistry, 29, 10219-10225.

33. Onufriev, A., Case, D. A. \& Ullmann, G. M. (2001). A novel view on the $\mathrm{pH}$ titration of biomolecules. Biochemistry, 40, 3413-3419.

34. Bashford, D. \& Gerwert, K. (1992). Electrostatic calculations of the $\mathrm{pK}$ a values of ionizable groups in bacteriorhodopsin. J. Mol. Biol. 224, 473-486.

35. Ondrechen, M. J., Clifton, J. G. \& Ringe, D. (2001). THEMATICS: a simple computational predictor of enzyme function from structure. Proc. Natl Acad. Sci. USA, 98, 12473-12478.

36. Rammelsberg, R., Huhn, G., Lübben, M. \& Gerwert, K. (1998). Bacteriorhodopsin's intramolecular proton-release pathway consists of a hydrogen-bonded network. Biochemistry, 37, 5001-5009.

37. Sampogna, R. V. \& Honig, B. (1994). Environmental effects on the protonation states of active site residues in bacteriorhodopsin. Biophys. J. 66, 1341-1352.

38. Brown, L. S., Bonet, L., Needleman, R. \& Lanyi, J. K. (1993). Estimated acid dissociation constants of the Schiff base, Asp-85 and Arg-82 during the bacteriorhodopsin photocycle. Biophys. J. 65, 124-130.

39. Balashov, S. P., Govindjee, R., Imasheva, E. S., Misra, S., Ebrey, T. G., Feng, Y. et al. (1995). The two pKa's of aspartate-85 and control of thermal isomerization and proton release in the arginine- 82 to lysine mutant of bacteriorhodopsin. Biochemistry, 35, $8820-8835$.

40. Balashov, S. P., Imasheva, E. S., Govindjee, R. \& Ebrey, T. G. (1996). Titration of aspartate-85 in bacteriorhodopsin: what it says about chromophore isomerization and protein release. Biophys. J. 70, 473-481.

41. Balashov, S. (2000). Protonation reactions and their 
coupling in bacteriorhodopsin. Biochim. Biophys. Acta, 1460, 75-94.

42. Steinhoff, H., Pfeiffer, M., Rink, T., Burlon, O., Kurz, M., Riesle, J. et al. (1999). Azide reduces the hydrophobic barrier of the bacteriorhodopsin proton channel. Biophys. J. 76, 2702-2710.

43. Schätzler, S., Dencher, N. A., Tittor, J., Oesterhelt, D., Yaniv-Checover, S., Nachliel, E. \& Gutman, M. (2003). Subsecond proton-hole propagation in bacteriorhodopsin. Biophys. J. 84, 671-686.

44. Scharnagl, C., Hettenkofer, J. \& Fischer, S. F. (1994). Proton release pathway in bacteriorhodopsin: molecular dynamics and electrostatic calculations. Int. J. Quantum Chem.: Quant. Biol. Symp. 21, 33-56.

45. Hendrickson, F. M., Burkard, F. \& Glaeser, R. M. (1998). Structural characterization of the L-to-M transition of the bacteriorhodopsin photocycle. Biophys. J. $75,1446-1454$.

46. Vonck, J., Han, B. G., Burkard, F., Perkins, G. A. \& Glaeser, R. M. (1994). Two progressive substates of the M-intermediate can be identified in glucoseembedded wild-type bacteriorhodopsin. Biophys. J. 67, 1173-1178.

47. Vonck, J. (1996). A three-dimensional difference map of the $\mathrm{N}$-intermediate in the bacteriorhodopsin photocycle: part of the f-helix tilts in the $\mathrm{M}$ to $\mathrm{N}$ transition. Biochemistry, 35, 5870-5878.

48. Dioumaev, A. K., Richter, H. T., Brown, L. S., Tanio, M., Tuzi, S., Saitô, H., Kimura, Y. et al. (1998). Existence of a proton transfer chain in bacteriorhodopsin: participation of Glu-194 in the release of protons to the extracellular surface. Biochemistry, 37, 2496-2506.

49. Balashov, S., Lu, M., Imasheva, E., Govindjee, R., Ebrey, T. \& Othersen, B. r. (1999). The proton release group of bacteriorhodopsin controls the rate of the final step of its photocycle at low ph. Biochemistry, 38, 2026-2039.

50. Schweiger, U., Tittor, J. \& Oesterhelt, D. (1994). Bacteriorhodopsin can function without a covalent linckage between retinal and protein. Biochemistry, 33, 535-541.

51. Friedman, N., Druckmann, S., Lanyi, J., Needleman, R., Lewis, A., Ottolenghi, M. \& Sheves, M. (1994). A covalent link between the chromophore and the protein backbone of bacteriorhodopsin is not required for forming a photochemically active pigment analogous to the wild type. Biochemistry, 33, 1971-1976.

52. Cao, Y., Váró, G., Klinger, A. L., Czajkowsky, D. M., Braiman, M. S., Needleman, R. \& Lanyi, J. K. (1993). Proton transfer from Asp-96 to the bacteriorhodopsin Schiff base is caused by a decrease of the $\mathrm{p} K_{\mathrm{a}}$ of Asp-
96 which follows a protein backbone conformational change. Biochemistry, 32, 1981-1990.

53. Cornell, W. D., Cieplak, P., Bayly, C. B., Gould, I. R., Mertz, K. M., Jr, Ferguson, D. M. et al. (1995). A second generation force field for the simulation of proteins, nucleic acids, and organic molecules. J. Am. Chem. Soc. 117, 5179.

54. Dillet, V., Van Etten, R. L. \& Bashford, D. (2000). Stabilization of charges and protonation states in the active site of the protein tyrosine phosphatases: a compuational study. J. Phys. Chem. B, 104, 11321-11333.

55. Demchuk, E., Genick, U. K., Woo, T. T. \& Donald, G. E. D. B. (2000). Protonation states and pH titration in the photocycle of photoactive yellow protein. Biochemistry, 39, 1100-1113.

56. Simonson, T. \& Perahia, D. (1995). Internal and interfacial dielectric properties of cytochrome $c$ from molecular dynamics in aqueous solution. Proc. Natl Acad. Sci. USA, 92, 1082-1086.

57. Bashford, D. (1997). An object-oriented programming suite for electrostatic effects in biological molecules. In Scientific Computing in Object-Oriented Parallel Environments In Lecture Notes in Computer Science, ISCOPE97 (Ishikawa, Y., Oldehoeft, R. R., Reynders, J. V. W. \& Tholburn, M., eds), vol. 1343, pp. 233-240, Springer, Berlin.

58. Smondyrev, A. M. \& Berkowitz, M. L. (1999). United atom force field for phospholipid membranes. Constant pressure molecular dynamics simulation of dppc/water system. J. Comp. Chem. 20, 531-545.

59. Berman, H. M., Westbrook, J., Feng, Z., Gilliland, G., Bhat, T. N., Weissig, H. et al. (2000). The protein data bank. Nucl. Acids Res. 28, 235-242.

60. Berendsen, H. J. C., Postma, J. P. M., van Gunsteren, W. F., DiNola, A. \& Haak, J. R. (1984). Molecular dynamics with coupling to an external bath. J. Chem. Phys. 81, 3684-3690.

61. MacKerell, A. D., Jr, Bashford, D., Bellott, M., Dunbrack, R. L., Jr, Evanseck, J. D., Field, M. J. et al. (1998). All-atom empirical potential for molecular modeling and dynamics studies of proteins. J. Phys. Chem. B, 102, 3586-3616.

62. Brown, L. S., Needleman, R. \& Lanyi, J. K. (1996). Interaction of proton and chloride transfer pathways in recombinant bacteriorhodopsin with chloride transport activity: implications for the chloride translocation mechanism. Biochemistry, 35, 16048-16054.

63. Chernavskii, D. S. \& Chernavskaya, N. M. (1999). Protein-Engine (in Russian), Janus-K, Moscow. 